\title{
Adjuvant Second-Dose Chemotherapy before Surgery for Patients with Locally Advanced Rectal Malignancy Is Not Beneficial: A Systematic Review and Meta-Analysis
}

\author{
Min Chen, Xue Song, Liang-zhou Chen, Lin Xu, Yi-pu Lu, and Jin-song Zhang \\ Department of General Surgery, Xiamen Traditional Chinese Medicine (TCM) Hospital Affiliated to Fujian University of TCM, \\ Xiamen 361009, China \\ Correspondence should be addressed to Min Chen; amadon@126.com
}

Received 16 April 2017; Revised 26 June 2017; Accepted 4 July 2017; Published 1 August 2017

Academic Editor: Alessandro Passardi

Copyright (c) 2017 Min Chen et al. This is an open access article distributed under the Creative Commons Attribution License, which permits unrestricted use, distribution, and reproduction in any medium, provided the original work is properly cited.

\begin{abstract}
Background. Preoperative chemoradiotherapy is the standard treatment for patients with locally advanced rectal cancer, although tumor responses vary widely; some patients may achieve a pathologic complete response rate (pCR) after chemoradiotherapy. Controversy exists with regard to the efficacy of different preoperative combination chemotherapy regimens and neoadjuvant chemoradiotherapy, compared with chemoradiotherapy alone. Methods. PubMed, the Cochrane Library, and Embase databases were searched for comparative studies of patients with locally advanced rectal cancer that were published between January 1991 and January 2016. Efficacies of different preoperative combination chemotherapy regimens and neoadjuvant chemoradiotherapy (group A) were compared with chemoradiotherapy alone (group B) in a meta-analysis using Review Manager v5.2. Results. Three prospective randomized controlled trials and two prospective nonrandomized controlled trials comprising 444 cases were eligible for analysis. No significant difference was detected in the rate of pCR (50/223, 22.4\% versus 35/223, 15.7\%; relative risk, RR: 1.42 [95\% confidence interval, CI: 0.97-2.09], $p=0.07$ ) between the two groups. The rate of tumor regression was similar for both groups (122/203, 60.1\% versus 111/203, 54.7\%; RR: 1.11 [95\% CI: 0.94-1.29], p=0.22). Conclusions. Adjuvant chemotherapy with preoperative chemoradiotherapy did not significantly improve the rate of pCR nor the rate of $\mathrm{T}$ and $\mathrm{N}$ downstaging.
\end{abstract}

\section{Background}

Colorectal cancer is one of the most common cancers worldwide and a leading cause of cancer death $[1,2]$. Previously, patients with colorectal cancer who underwent operative resection had a high rate of local recurrence. However, with the introduction of new operative procedures for total mesorectal excision (TME), the envelope of lymphovascular fatty tissue and the mesorectum [3] was completely excised, leading to a significant decrease in the local recurrence rate of colorectal cancer $[4,5]$. Besides improvements in surgical technique, adjuvant therapy regimens provided excellent complementary therapy to reduce local recurrence rates of colorectal cancer.

Preoperative radiotherapy combined with TME can cut the 10 -year local recurrence rate by more than half-relative to that with TME alone [6]. Locally advanced rectal cancer refers to clinical T3 N0 (or any-T, but N1-N2 disease), and approximately $70 \%$ of rectal cancers without metastasis are locally advanced [7], which emphasizes the necessity of neoadjuvant chemotherapy management of locally advanced rectal cancers. Although preoperative radiotherapy can significantly decrease local recurrence rates, it cannot effectively downstage the tumor itself, regardless of whether shortcourse [8-10] or conventionally fractionated radiotherapy with delayed TME is adopted as the treatment strategy [11]. As the combined effect of radiotherapy and chemotherapy has been noted experimentally [12], systemic chemotherapy was introduced in combination with preoperative radiotherapy to constitute neoadjuvant chemoradiotherapy.

Comparison of outcomes between patients receiving chemoradiotherapy and those receiving preoperative 
radiotherapy alone indicated that the local recurrence rate was significantly lower in the preoperative chemoradiotherapy group [13]. Moreover, the local recurrence rate of colorectal cancer was significantly decreased in the preoperative, as compared with the postoperative, chemoradiotherapy group [14]. Worldwide, chemoradiotherapy followed by TME has become the standard treatment for locally advanced rectal cancer. A number of recent studies have explored modified neoadjuvant chemoradiotherapy regimens, wherein a common strategy involves adjuvant chemotherapy either before or after chemoradiotherapy such that patients receive a sufficient therapeutic radiation dose as well as the intensity of chemotherapy. Despite being studied in several trials, conflicting results have been reported with these novel treatment regimens, and only some reports noted that additional chemotherapy increased the rate of pathologic complete response (pCR) $[15,16]$.

This meta-analysis of comparative trials was conducted to evaluate the effect of adjuvant chemotherapy in combination with chemoradiotherapy, as compared with chemoradiotherapy alone, on $\mathrm{pCR}$ in locally advanced rectal cancer.

\section{Methods}

2.1. Search Strategy. A comprehensive literature search was conducted in January 2016. Studies published between January 1991 and prior to January 2016 were identified in the following databases: Embase, PubMed, and the Cochrane Library, without restrictions of the region, publication type, or language. The following $\mathrm{MeSH}$ terms and their combinations were searched [Title/Abstract]: locally advanced, rectal/rectum, cancer/tumour/tumor/neoplasm, and chemoradiation/radiochemotherapy/chemoradiotherapy. Furthermore, the related "Articles" function in Review Manager was used to broaden the search. The computerized search was supplemented with a manual examination of reference lists of all retrieved studies, review articles, and conference abstracts. If multiple reports described the same populations, the most recent or complete report was used.

The full electronic search strategy used the following search terms for PubMed: (locally advanced) AND (rectal) OR rectum AND (cancer) OR (tumour) OR tumor) OR neoplasm OR "Rectal Neoplasms" [MeSH] AND (chemoradiation) OR radiochemotherapy) OR chemoradiotherapy) OR “Chemoradiotherapy” [MeSH].

2.2. Inclusion and Exclusion Criteria. All randomized controlled trials (RCTs) available as full-text or conference abstracts, prospective comparative studies that compared the efficacy of combined adjuvant chemotherapy and chemoradiotherapy with chemoradiotherapy alone in locally advanced rectal cancer, and studies with rate of $\mathrm{pCR}$ as one of the outcomes were included. Retrospective and noncomparative studies, editorials, letters to the editor, review articles, case reports, or preclinical studies were excluded.

2.3. Data Collection. The predefined information from the included studies was extracted and summarized independently by two investigators (JS Zhang and YP Lu). Information about the characteristics of the study population and relevant outcomes was recorded. Disagreements pertaining to data abstraction were resolved through discussion and, if unresolved, arbitrated by a third investigator (X Song) to reach consensus.

The incidence of pCR was selected as the primary outcome. pCR refers to the absence of tumor cells in the surgical specimen, including both at the primary tumor site and regional lymph nodes (ypT0N0). The secondary outcome was the downstaging effect, which was defined as the rate of posttreatment reduction of pathological stage (including both $\mathrm{T}$ and/or $\mathrm{N}$ categories) as compared with the pretreatment clinical stage.

Guidelines of the Cochrane Collaboration were used to assess the quality of the RCTs, [17] which were evaluated by six categories: sequence generation, allocation concealment, blinding, incomplete outcome data, selective outcome reporting, and other biases. Blinding was deemed to confer a low risk of bias if outcome assessors were blinded to the treatment information. The modified Newcastle-Ottawa scale was used to assess the quality of the prospective nonRCTs across three factors: patient selection, comparability of study groups, and assessment of outcome. A score of 0-9 allocated stars was used to evaluate the quality of nonrandomized controlled trials. RCTs and studies that received six or more stars were considered to be of high quality and were included in the meta-analysis.

2.4. Statistical Analysis. All statistical analyses were conducted using Review Manager v5.2 (Cochrane Collaboration, Oxford, England). A fixed model was used if there was no evidence of heterogeneity; otherwise, a random-effects model was used. Statistical heterogeneity between studies was assessed using the chi-squared test, with significance set at $p<0.10$ and heterogeneity quantified using the $I^{2}$ statistic. Relative risk (RR) was calculated for each trial from the number of evaluable patients, and all results were reported with 95\% confidence intervals (CI). The $p$ value for the overall effect was calculated using the $z$-test, with significance set at $p<0.05$. A sensitivity analysis was conducted. Publication bias was not assessed due to the small number of trials.

\section{Results}

3.1. Trial Characteristics. The trial selection process is shown in Figure 1. Three RCTs and two nonrandomized trials that met the predefined inclusion criteria were eligible for final analysis. These studies included 223 patients who received preoperative adjuvant chemotherapy and chemoradiotherapy (group A) as well as 223 patients who received preoperative chemoradiotherapy alone (group B). The characteristics of these five studies are shown in Table 1. The risks of biases of three RCTs and the two nonrandomized studies are shown in Tables 2 and 3, respectively.

\subsection{Results of Meta-Analysis}

3.2.1. Pathologic Complete Response. All of the included studies reported pCR rates that were 22.4\% (50/223) and 


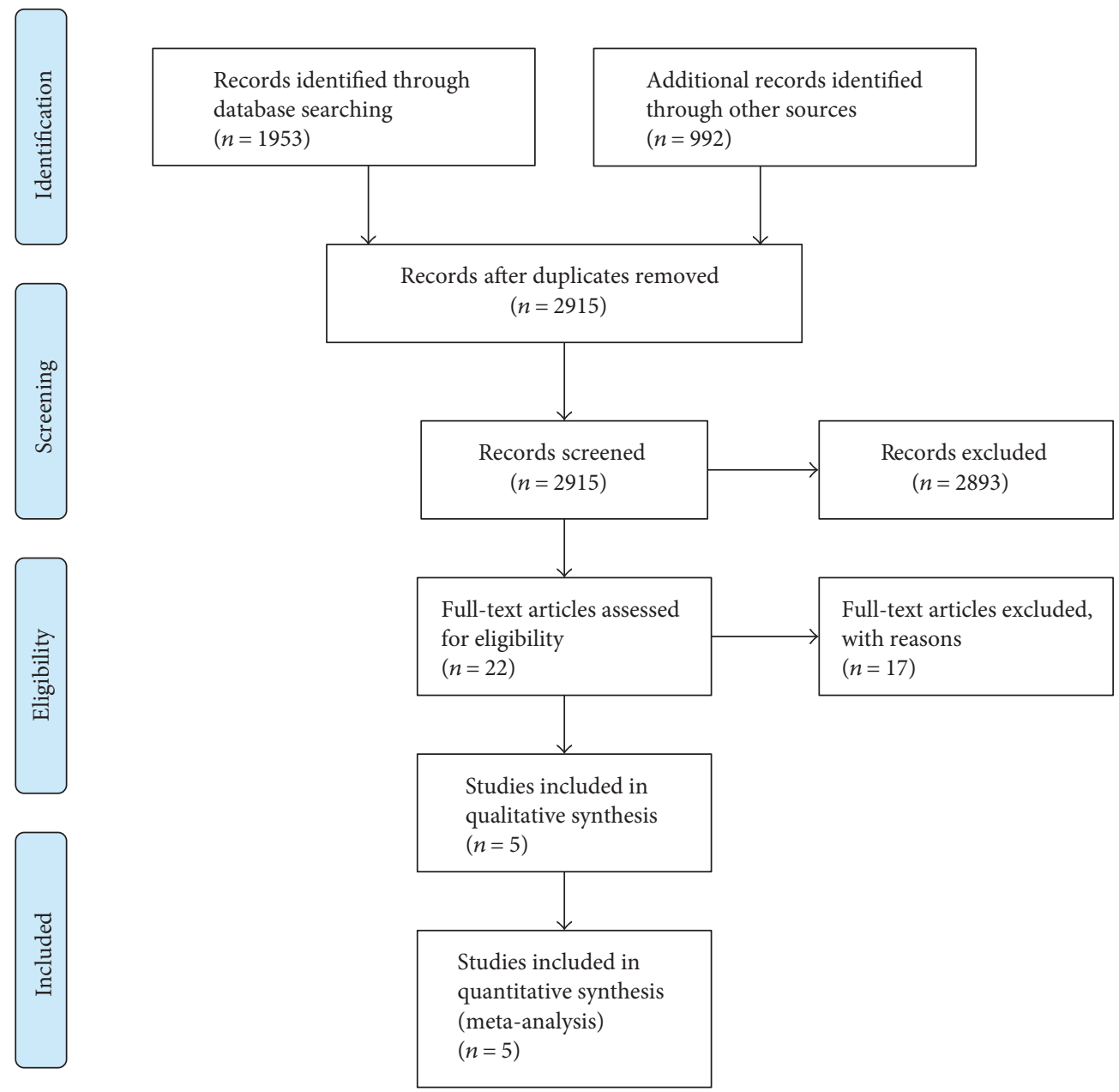

FIGURE 1: Flow diagram of trial selection process for inclusion in the meta-analysis.

15.7\% (35/223) for group A and group B, respectively. No significant difference was detected in the rate of pCR (RR: 1.42 [95\% CI: 0.97-2.09], $p=0.07$ ) between the two groups (Figure 2).

3.2.2. Downstaging. Rates of downstaging were reported in four of the included studies and were $60.1 \%(122 / 203)$ in group A, but $54.7 \%(111 / 203)$ in group B. The addition of chemotherapy to chemoradiotherapy did not significantly improve the rate of downstaging (RR: 1.11 [95\% CI: 0.941.29 ], $p=0.22$; Figure 3).

3.3. Sensitivity Analysis and Publication Bias. Sensitivity analysis was conducted to determine the significance of each set of results. Three RCTs were included in the sensitivity analysis (Table 4), but there was no statistically significant change with regard to the outcome. Publication bias was not assessed due to the small number of trials.

\section{Discussion}

Chemoradiotherapy followed by TME in combination with postoperative adjuvant chemotherapy is a standard treatment for locally advanced rectal cancer. A limitation of this treatment is the need for patient compliance to postoperative adjuvant chemotherapy. Less than $50 \%$ of patients usually receive postoperative adjuvant chemotherapy [18]. A common therapeutic strategy is to add chemotherapy before or after chemoradiotherapy to improve compliance with chemotherapy to ensure patients receive a sufficient therapeutic dose and intensity of chemotherapy.

Patients with colorectal cancer respond to chemoradiotherapy differently; some patients may achieve a pCR after chemoradiotherapy, which suggests better prognostic outcomes. Maas et al. reported that the 5-year crude diseasefree survival (DFS) was $83.3 \%$ in patients with pCR $(61 / 419$ patients had disease recurrence) and $65.6 \%$ in those without pCR $(p<0.0001)$ [19] Kuo et al. reported that the rate of recurrence was significantly different between the pCR and residual tumor groups (5.6\% versus $31.1 \% ; p=0.002)$. The 5 -year DFS was significantly better in the pCR group than in the residual tumor group (93\% versus $66 \% ; p=0.0045$ ) [20]. Chari et al. reported that, during the follow-up period, 39 of the 43 patients remained alive (median follow-up 25 months), and only 1 of the 11 patients with complete histologic response developed recurrent disease. Six of the 32 


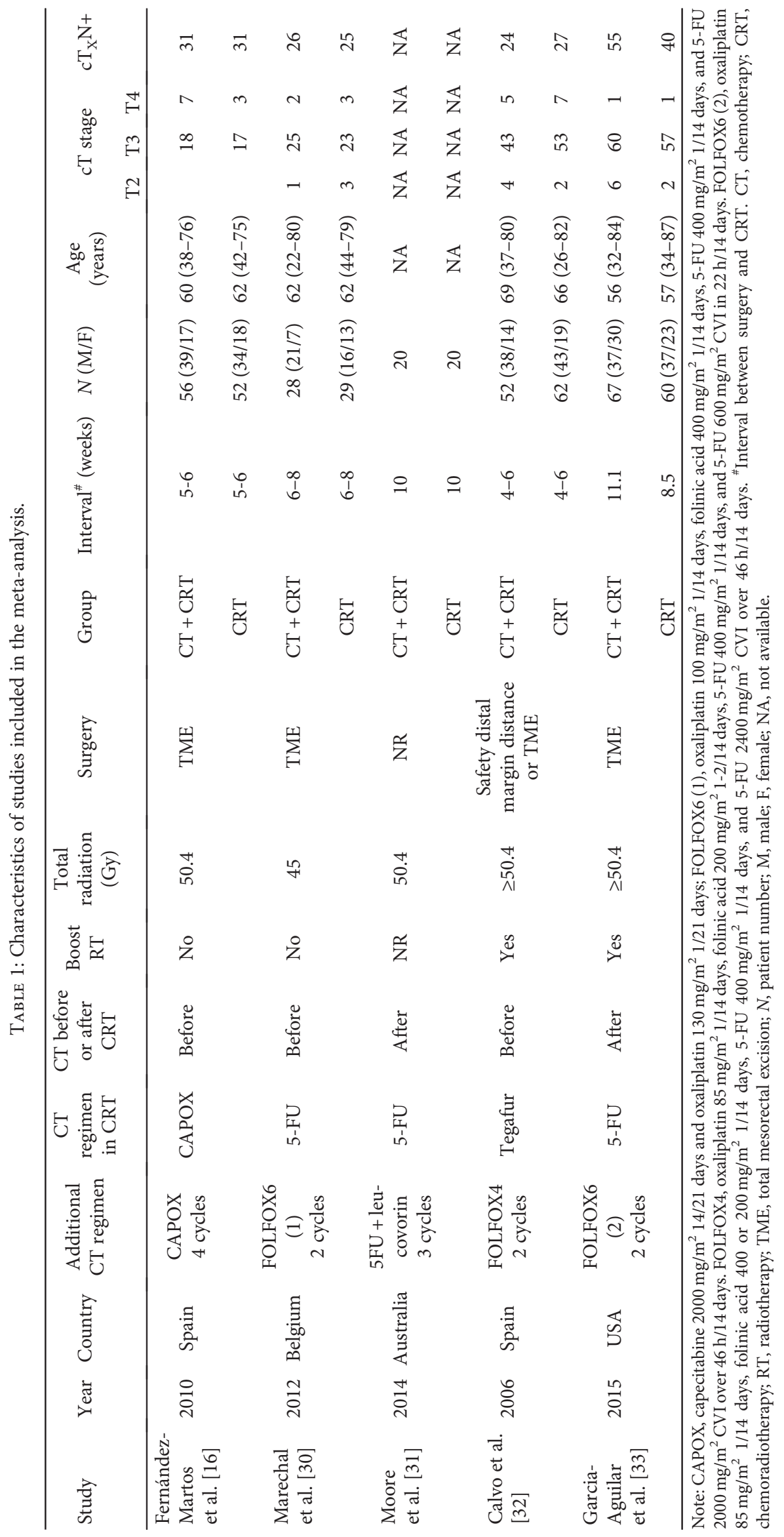


TABLE 2: Risk of bias in the prospective randomized controlled studies.

\begin{tabular}{lcccccc}
\hline Study & $\begin{array}{c}\text { Adequate random } \\
\text { sequence generation }\end{array}$ & $\begin{array}{c}\text { Allocation } \\
\text { concealment }\end{array}$ & $\begin{array}{c}\text { Blinding of participants } \\
\text { and personnel }\end{array}$ & $\begin{array}{c}\text { Incomplete } \\
\text { outcome data }\end{array}$ & $\begin{array}{c}\text { Selective outcome } \\
\text { reporting }\end{array}$ & $\begin{array}{c}\text { Other } \\
\text { biases }\end{array}$ \\
\hline $\begin{array}{l}\text { Fernández-Martos } \\
\text { et al. [16] }\end{array}$ & Yes & No & Yes & Yes & Yes & Yes \\
Marechal et al. [30] & Yes & No & Yes & Yes & Yes & Yes \\
Moore et al. [31] & No & No & Yes & Yes & Yes & Yes \\
\hline
\end{tabular}

TABLE 3: Risk of bias in the nonrandomized studies using modified Newcastle-Ottawa scale.

\begin{tabular}{|c|c|c|c|c|c|c|c|c|}
\hline \multirow[t]{2}{*}{ Study } & \multicolumn{3}{|c|}{ Selection } & \multicolumn{2}{|c|}{ Comparability } & \multicolumn{2}{|c|}{ Outcome } & \multirow[t]{2}{*}{ Quality score } \\
\hline & $\begin{array}{l}\text { Assign for } \\
\text { treatment }^{\triangle}\end{array}$ & $\begin{array}{l}\text { Representative } \\
\text { treatment } \\
\text { group }\end{array}$ & $\begin{array}{l}\text { Representative } \\
\text { reference } \\
\text { group }\end{array}$ & $\begin{array}{c}\text { Comparable } \\
\text { for } 1,2,3,4 \text {, } \\
\text { and } 5^{*}\end{array}$ & $\begin{array}{c}\text { Comparable } \\
\text { for } 6,7,8, \\
\text { and } 9^{*}\end{array}$ & $\begin{array}{l}\text { Assessment } \\
\text { of outcome }\end{array}$ & $\begin{array}{l}\text { Adequate } \\
\text { follow-up }\end{array}$ & \\
\hline Calvo et al. [32] & No & Yes & Yes & 1,2 , and 3 & 6,7 , and 8 & Yes & Yes & $\star \star \star \star \star \star \star$ \\
\hline $\begin{array}{l}\text { Garcia-Aguilar } \\
\text { et al. [33] }\end{array}$ & No & Yes & Yes & $1,2,3$, and 4 & 6,7 , and 8 & Yes & Yes & 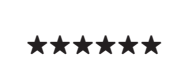 \\
\hline
\end{tabular}

Comparability variables: 1 , age; 2 , gender; 3 , TN; 4, total mesorectal excision; 5 , radial margin status; 6 , distance from anal verge; 7 , radiotherapy technique; 8 , total dose of radiotherapy; and 9 , adjuvant chemotherapy. ${ }^{*}$ If all characteristics were comparable, two stars; if $>2$ characteristics were comparable, one star; if $<2$ characteristics were comparable, no star. ${ }^{\triangle}$ The article provided the details of criteria for adequate random assignment.

patients with residual disease (two with positive nodes) developed metastatic disease during follow-up (time to diagnosis: median 10 months, range 3-15 months) [21].

Approximately $15 \%-27 \%$ of patients with colorectal cancer can achieve a complete response; however, this percentage is not considered indicative of successful treatment, and increasing this proportion remains a major clinical challenge [19]. Therefore, we conducted a meta-analysis of comparative trials to evaluate the effect of adjuvant chemotherapy administered in combination with chemoradiotherapy on pCR in locally advanced rectal cancer.

The current review addressed the question whether adjuvant chemotherapy with preoperative chemoradiotherapy further improved the rate of pCR. We identified three RCTs and two nonrandomized trials that compared preoperative chemotherapy plus chemoradiotherapy against chemoradiotherapy alone in locally advanced rectal cancer. A metaanalysis of their results showed that additional chemotherapy did not significantly increase the rate of $\mathrm{pCR}$, compared with chemoradiotherapy alone. Furthermore, adjuvant chemotherapy with preoperative chemoradiotherapy did not improve the rate of downstaging. However, another aspect was that this therapeutic modality did not increase the rate of grade 3-4 toxicity.

One study indicated that if preoperative radiotherapy is to be effective, then the biologically equivalent dose should be at least $20 \mathrm{~Gy}$ [22]. Furthermore, another study reported that different doses of preoperative radiotherapy ( $\geq 30 \mathrm{~Gy}$ ) could have anticancer biological effects [23]. All five studies were reviewed with a biologically equivalent dose $>45 \mathrm{~Gy}$, although the doses and fractionations of radiotherapy were different and radiotherapy regimens were identical in both groups in all of the studies. According to the results of our meta-analysis, we infer that radiotherapy doses cannot influence changes in the rate of pCR and T/N downstaging.

Boost radiation was undertaken in two studies and, despite the dose and regimen of the radiotherapy being different, results of these studies showed that adjuvant chemotherapy with chemoradiotherapy significantly improved the rate of pCR. However, no benefit in the rate of pCR was conferred with the addition of a boost radiotherapy dose $[24,25]$. This difference may be attributed to the adjuvant chemotherapy administered in combination with the boost radiation. A meta-analysis of the results of these two studies using the Mantel-Haenszel random-effects model did not reveal a significant conclusion. Thus, the effect on the rate of pCR of such adjuvant chemotherapy and boost radiotherapy dose in combination with chemoradiotherapy remains uncertain. Indeed, it does not appear to be clinically sound.

A meta-analysis has shown that an interval longer than 6 to 8 weeks between chemoradiotherapy and surgery could significantly improve pCR [26]. The interval between chemoradiotherapy and surgery in four of the five studies was consistent, except for a study by Garcia-Aguilar (11.1 weeks versus 8.5 weeks). However, in this study, the interval in both groups was longer than 6 to 8 weeks, and the difference in intervals did not affect the overall results of the meta-analysis.

Three trials used induction chemotherapy before chemoradiotherapy. Induction chemotherapy involves the application of chemotherapy with sufficient dose and intensity. Chau and colleagues reported that induction with capecitabine and oxaliplatin before chemoradiotherapy and TME may increase the tumor response rate up to $97 \%$. Moreover, after induction with capecitabine/oxaliplatin, the radiologic response rate was $88 \%$ [27]. 


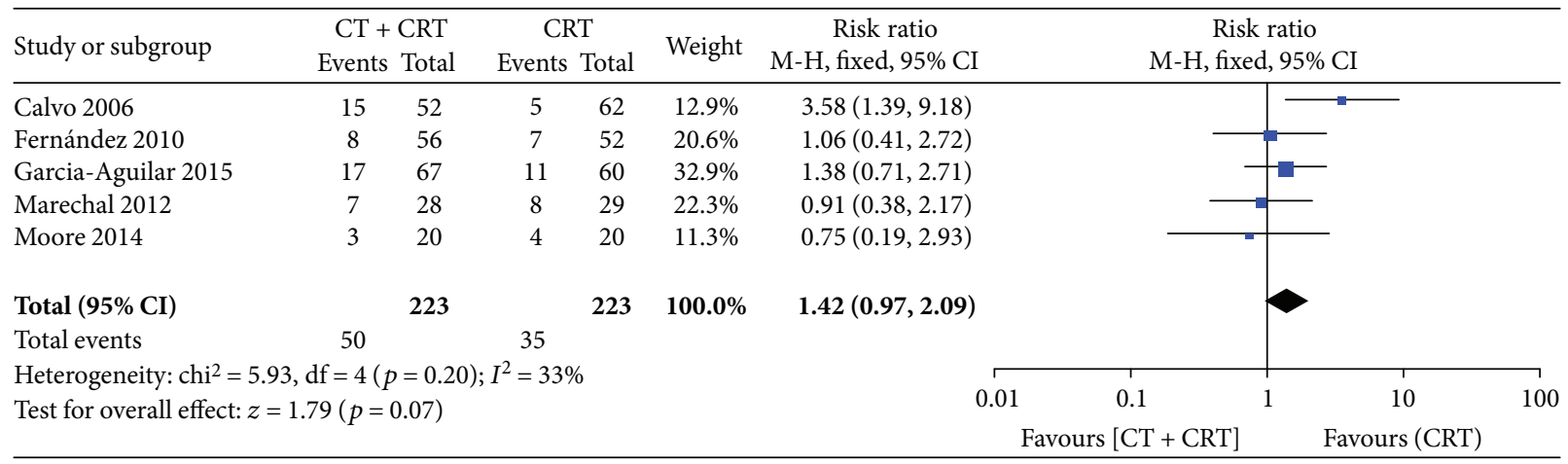

Figure 2: Forest plot for pCR. A Mantel-Haenszel fixed-effects model was used for meta-analysis. Risk ratios are shown with 95\% confidence interval.

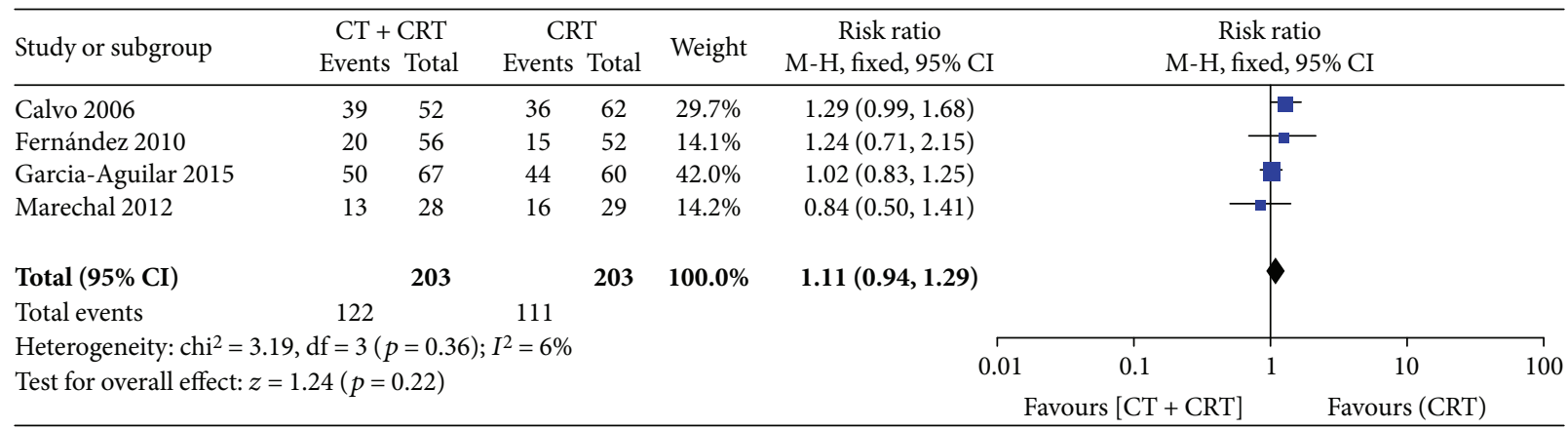

FIGURE 3: Forest plot for downstaging. A Mantel-Haenszel fixed-effects model was used for meta-analysis. Risk ratios are shown with 95\% confidence interval.

TABLE 4: Comparison of sensitivity analysis of pCR in the three RCTs.

\begin{tabular}{|c|c|c|c|c|c|c|c|c|}
\hline Outcomes of interest & Number of studies & $\mathrm{CT}+\mathrm{CRT}$ number & CRT number & RR (95\% CI) & $p$ value & \multicolumn{3}{|c|}{ Study heterogeneity } \\
\hline & & & & & & $\mathrm{df}$ & $I^{2}(\%)$ & $p$ value \\
\hline pCR & 3 & 104 & 101 & $0.93(0.52-1.66)$ & 0.81 & 2 & 0 & 0.92 \\
\hline
\end{tabular}

We included these three studies in the meta-analysis and found that induction chemotherapy before chemoradiotherapy did not significantly improve the overall pCR. Two trials used chemotherapy after chemoradiotherapy. The rationale of this strategy is that the tumor response to chemoradiotherapy is time-dependent, whereas shorter intervals may interrupt ongoing tumor necrosis [28]. Moreover, using chemotherapy after chemoradiotherapy allowed the administration of sufficient dose and the intensity of chemotherapy and, thus, prolonged the interval between chemoradiotherapy and surgery; this allowed enough time for a tumor response to achieve pCR. The same conclusion can be drawn from these two studies: adjuvant chemotherapy after chemoradiotherapy did not improve the pCR significantly.

Three studies used chemotherapy (which contained oxaliplatin) before chemoradiotherapy. Combination therapy with oxaliplatin could enhance the antitumor effect of 5-fluorouracil compared with 5-fluorouracil monotherapy; moreover, it could function as a potent radiosensitizing agent [29]. Finally, these three studies showed that the addition of oxaliplatin-containing chemotherapy to chemoradiotherapy did not improve the pCR significantly.

The main limitation of our review is that only five published manuscripts form the basis of its meta-analysis; moreover, two of the five studies were not RCTs. This may have potentially increased the risk of bias due to inadequate randomization. One of the RCTs included was only available as a conference abstract, which does not provide complete data, and was, therefore, not considered a scientific evidenced-based study. Furthermore, the regimen of adjuvant chemotherapy and chemoradiotherapy as well as the interval between chemoradiotherapy and surgery differed across the five studies, which may have resulted in an underestimation of the overall therapeutic efficacy. Finally, the number of patients evaluated is small (only 223 patients in each arm), which may be inadequate to identify distinct differences in the rate of pCR. Therefore, another metaanalysis that includes other studies with more data will be required in the future to elucidate further on the differences between the studied chemotherapeutic and chemoradiotherapeutic regimens. 


\section{Conclusions}

The addition of a second dose of chemotherapy to the primary chemoradiotherapy regimen before TME in locally advanced rectal cancer did not change the rate of pCR or that of $\mathrm{T}$ and $\mathrm{N}$ downstaging. Given the inherent limitations of this meta-analysis, additional large-scale RCTs that evaluate the outcomes and safety with adjuvant chemotherapy and chemoradiotherapy are warranted.

\section{Conflicts of Interest}

The authors declare that they have no competing interests.

\section{References}

[1] R. Siegel, D. Naishadham, and A. Jemal, "Cancer statistics, 2013,” CA: A Cancer Journal for Clinicians, vol. 63, pp. 11-30, 2013.

[2] J. Ferlay, E. Steliarova-Foucher, J. Lortet-Tieulent et al., "Cancer incidence and mortality patterns in Europe: estimates for 40 countries in 2012," European Journal of Cancer, vol. 49, pp. 1374-1403, 2013.

[3] R. J. Heald, E. M. Husband, and R. D. Ryall, "The mesorectum in rectal cancer surgery-the clue to pelvic recurrence?" The British Journal of Surgery, vol. 69, pp. 613-616, 1982.

[4] W. E. Enker, N. Merchant, A. M. Cohen et al., "Safety and efficacy of low anterior resection for rectal cancer: 681 consecutive cases from a specialty service," Annals of Surgery, vol. 230, pp. 544-554, 1999.

[5] C. A. Maurer, P. Renzulli, C. Kull et al., "The impact of the introduction of total mesorectal excision on local recurrence rate and survival in rectal cancer: long-term results," Annals of Surgical Oncology, vol. 18, pp. 1899-1906, 2011.

[6] W. van Gijn, C. A. Marijnen, I. D. Nagtegaal et al., "Preoperative radiotherapy combined with total mesorectal excision for resectable rectal cancer: 12-year follow-up of the multicentre, randomised controlled TME trial," The Lancet Oncology, vol. 12, pp. 575-582, 2011.

[7] A. Vignali and P. De Nardi, "Multidisciplinary treatment of rectal cancer in 2014: where are we going?" World Journal of Gastroenterology, vol. 20, pp. 11249-11261, 2014.

[8] Y. Francois, C. J. Nemoz, J. Baulieux et al., "Influence of the interval between preoperative radiation therapy and surgery on downstaging and on the rate of sphincter-sparing surgery for rectal cancer: the Lyon R90-01 randomized trial," Journal of Clinical Oncology, vol. 17, pp. 2396-2402, 1999.

[9] C. A. M. Marijnen, I. D. Nagtegaal, E. Klein Kranenbarg et al., "No downstaging after short-term preoperative radiotherapy in rectal cancer patients," Journal of Clinical Oncology, vol. 19, pp. 1976-1984, 2001.

[10] W. Graf, M. Dahlberg, M. M. Osman, L. Holmberg, L. Påhlman, and B. Glimelius, "Short-term preoperative radiotherapy results in down-staging of rectal cancer: a study of 1316 patients," Radiotherapy and Oncology, vol. 43, pp. 133137, 1997.

[11] A. Horn, J. F. Halvorsen, and O. Dahl, "Preoperative radiotherapy in operable rectal cancer," Diseases of the Colon and Rectum, vol. 33, pp. 823-828, 1990.

[12] J. E. Byfield, P. Calabro Jones, I. Klisak, and F. Kulhanian, "Pharmacologic requirements for obtaining sensitization of human tumor cells in vitro to combined 5-fluorouracil or ftorafur and X rays," International Journal of Radiation Oncology Biology Physics, vol. 8, pp. 1923-1933, 1982.

[13] W. Ceelen, K. Fierens, Y. Van Nieuwenhove, and P. Pattyn, "Preoperative chemoradiation versus radiation alone for stage II and III resectable rectal cancer: a systematic review and meta-analysis," International Journal of Cancer, vol. 124, pp. 2966-2972, 2009.

[14] R. Sauer, H. Becker, W. Hohenberger et al., "Preoperative versus postoperative chemoradiotherapy for rectal cancer," The New England Journal of Medicine, vol. 351, pp. 17311740, 2004.

[15] K. H. Lee, J. S. Kim, J. Y. Kim, J. Jang, C. N. Kim, and W. J. Choi, "Prospective study of additional chemotherapy to preoperative chemoradiotherapy with capecitabine; increased complete response rate and acceptable toxicity," Colorectal Disease, vol. 15, p. 84, 2013.

[16] C. Fernández-Martos, C. Pericay, J. Aparicio et al., "Phase II, randomized study of concomitant chemoradiotherapy followed by surgery and adjuvant capecitabine plus oxaliplatin (CAPOX) compared with induction CAPOX followed by concomitant chemoradiotherapy and surgery in magnetic resonance imaging-defined, locally advanced rectal cancer: Grupo cancer de recto 3 study," Journal of Clinical Oncology, pp. 859-865, 2010.

[17] J. P. T. Higgins and D. G. Altman, "Assessing risk of bias in included studies," in In Cochrane Handbook for Systematic Reviews of Interventions, pp. 187-241, John Wiley \& Sons, Ltd, 2008.

[18] J. F. Bosset, L. Collette, G. Calais et al., "Chemotherapy with preoperative radiotherapy in rectal cancer," The New England Journal of Medicine, vol. 355, pp. 1114-1123, 2006.

[19] M. Maas, P. J. Nelemans, V. Valentini et al., "Long-term outcome in patients with a pathological complete response after chemoradiation for rectal cancer: a pooled analysis of individual patient data," The Lancet Oncology, vol. 11, pp. 835-844, 2010.

[20] L. J. Kuo, M. C. Liu, J. J. Jian et al., "Is final TNM staging a predictor for survival in locally advanced rectal cancer after preoperative chemoradiation therapy?," Annals of Surgical Oncology, vol. 14, pp. 2766-2772, 2007.

[21] R. S. Chari, D. S. Tyler, M. S. Anscher et al., "Preoperative radiation and chemotherapy in the treatment of adenocarcinoma of the rectum," Annals of Surgery, vol. 221, pp. 778-787, 1995.

[22] J. P. Ciria, M. Eguiguren, S. Cafiero et al., "Could preoperative short-course radiotherapy be the treatment of choice for localized advanced rectal carcinoma?," Reports of Practical Oncology and Radiotherapy, vol. 20, no. 1, pp. 1-11, 2014.

[23] Adjuvant radiotherapy for rectal cancer: a systematic overview of 8,507 patients from 22 randomised trials," Lancet, vol. 358, pp. 1291-1304, 2001.

[24] A. Jakobsen, J. Ploen, T. Vuong, A. Appelt, J. Lindebjerg, and S. R. Rafaelsen, "Dose-effect relationship in chemoradiotherapy for locally advanced rectal cancer: a randomized trial comparing two radiation doses," International Journal of Radiation Oncology, Biology, Physics, vol. 84, pp. 949954, 2012.

[25] A. L. Appelt, I. R. Vogelius, J. Ploen et al., "Long-term results of a randomized trial in locally advanced rectal cancer: no benefit from adding a brachytherapy boost," In International Journal of Radiation Oncology Biology Physics, pp. 110-118, 2014. 
[26] F. Petrelli, G. Sgroi, E. Sarti, and S. Barni, "Increasing the interval between neoadjuvant chemoradiotherapy and surgery in rectal cancer: a meta-analysis of published studies," Annals of Surgery, 2013.

[27] I. Chau, G. Brown, D. Cunningham et al., "Neoadjuvant capecitabine and oxaliplatin followed by synchronous chemoradiation and total mesorectal excision in magnetic resonance imaging-defined poor-risk rectal cancer," Journal of Clinical Oncology, vol. 24, pp. 668-674, 2006.

[28] A. Habr-Gama, R. O. Perez, I. Proscurshim et al., "Interval between surgery and neoadjuvant chemoradiation therapy for distal rectal cancer: does delayed surgery have an impact on outcome?," International Journal of Radiation Oncology, Biology, Physics, vol. 71, pp. 1181-1188, 2008.

[29] C. Aschele, M. L. Friso, S. Pucciarelli et al., "A phase I-II study of weekly oxaliplatin, 5-fluorouracil continuous infusion and preoperative radiotherapy in locally advanced rectal cancer," Annals of Oncology, vol. 16, pp. 1140-1146, 2005.

[30] R. Marechal, B. Vos, M. Polus et al., "Short course chemotherapy followed by concomitant chemoradiotherapy and surgery in locally advanced rectal cancer: a randomized multicentric phase II study," Annals of Oncology, vol. 23, pp. 1525-1530, 2012.

[31] J. Moore, P. Hewett, E. Murphy et al., "Preoperative chemotherapy following neoadjuvant chemoradiotherapy for rectal cancer does not improve pCR rates," Colorectal Disease, vol. 16, pp. 15-16, 2014.

[32] F. A. Calvo, F. J. Serrano, J. A. Diaz-Gonzalez et al., "Improved incidence of pT0 downstaged surgical specimens in locally advanced rectal cancer (LARC) treated with induction oxaliplatin plus 5-fluorouracil and preoperative chemoradiation," Annals of Oncology, vol. 17, pp. 1103-1110, 2006.

[33] J. Garcia-Aguilar, O. S. Chow, D. D. Smith et al., "Effect of adding mFOLFOX6 after neoadjuvant chemoradiation in locally advanced rectal cancer: a multicentre, phase 2 trial," The Lancet Oncology, vol. 16, pp. 957-966, 2015. 


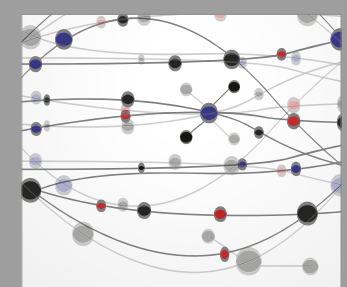

The Scientific World Journal
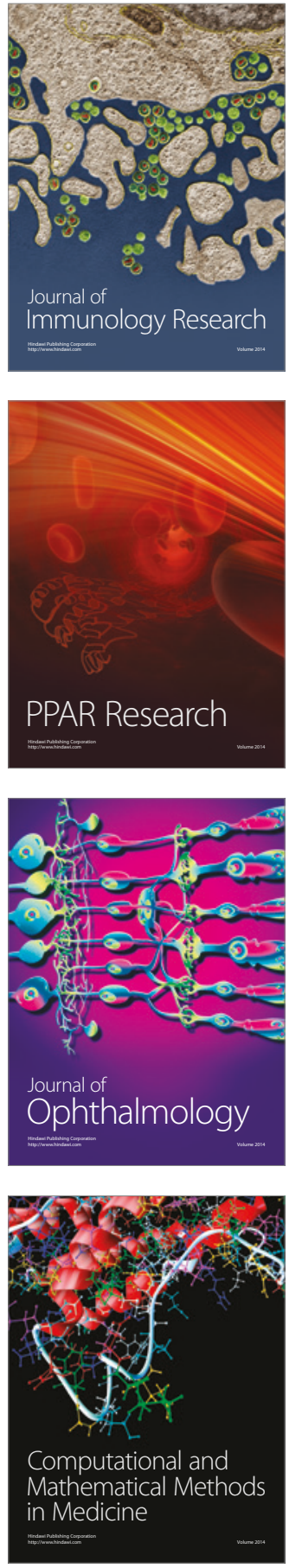

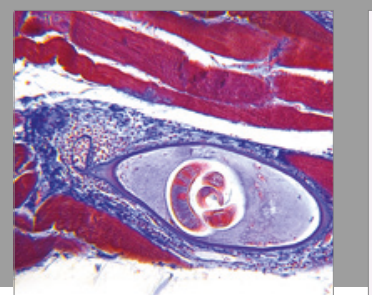

Gastroenterology Research and Practice
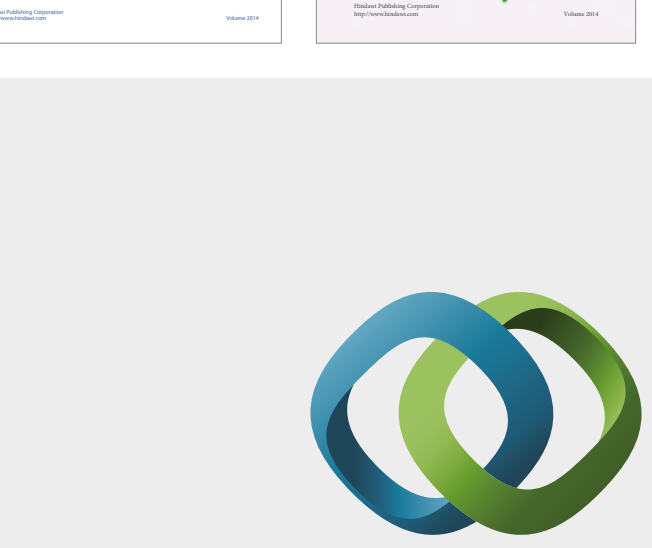

\section{Hindawi}

Submit your manuscripts at

https://www.hindawi.com
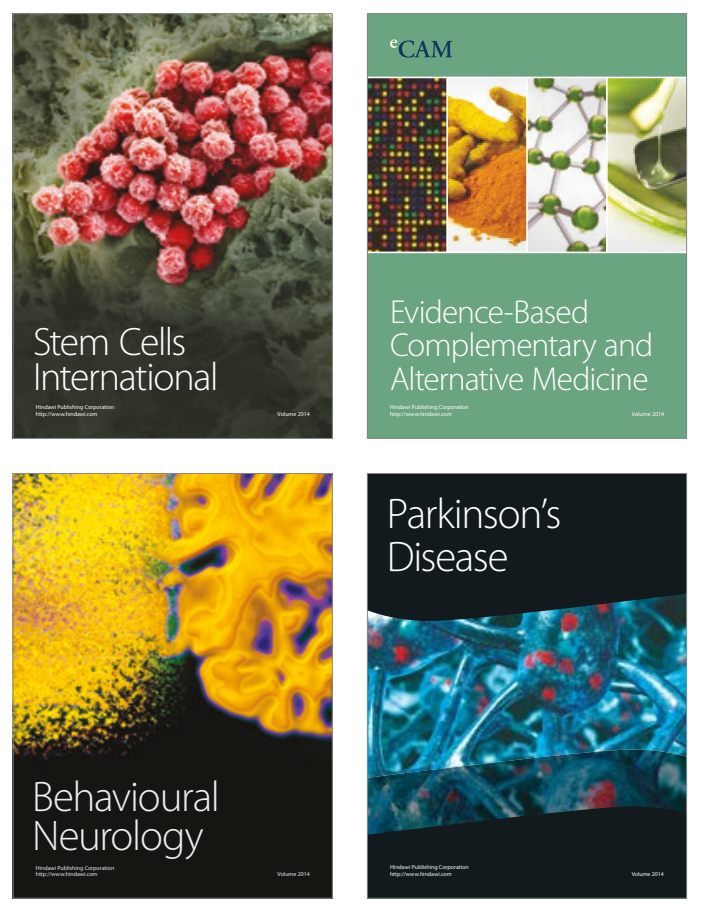
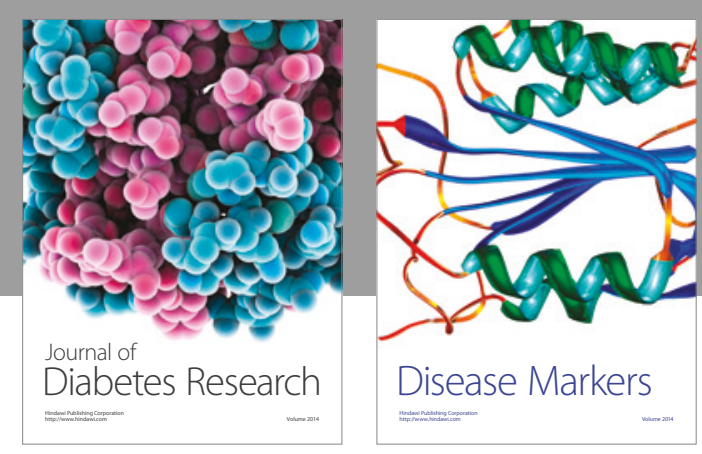

Disease Markers
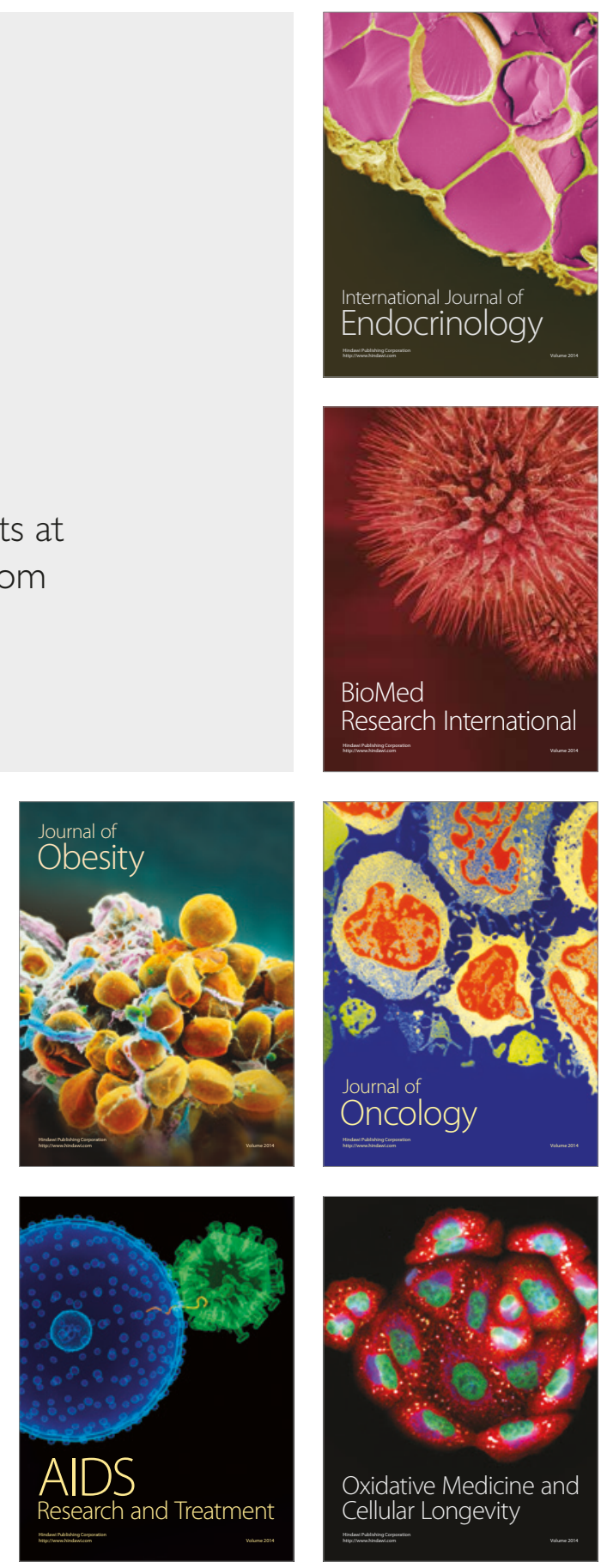